\title{
Assessment of serum biomarkers of oxidative stress in patients with chronic renal failure
}

\author{
Dr. Sarala Devi Tenepalli ${ }^{1}$, Dr. Karunasree Yerrajwala ${ }^{2}$ \\ ${ }^{I}$ Associate Professor, Department of Biochemistry, MNR Medical College and Hospital, Sangareddy, \\ Telangana. \\ ${ }^{2}$ Professor, Department of Biochemistry, MNR Medical College and Hospital, Sangareddy, Telangana.
}

\begin{abstract}
Chronic renal failure (CRF) is becoming a major health challenges among worldwide. Incidence of the disease is alarming, because an insufficient therapeutic methods. Based on the estimated glomerular filtration rate (eGFR), the CRF is being classifying in to numerous phases. Recent guidelines classified the severity of CRF into 5 phases. First stage is being the mildest and fifth stage being a severe illness with less lifeexpansion.The present study was designed to assess the link between CRF and oxidative stress markers, urea, creatinine and serum creatinine levels in Patients with chronic renal failure.A total fifty CRF patients and fifty healthy control subjects were considered, based on the eGFR values patients were assigned to five phases. The results indicating that serum lipid peroxidation was significantly increased in all the phases of CRF patients when compared to the controls. Serum superoxide dismutase mean values are not showing any variation in first phase but, in later phases values were graduallydecreased as compared to control subjects. The study reinforce the possibility that antioxidant supplementation is helpful to CRF patents. It is extremely important to know oxidative stress in CRF patient right from the time of diagnosis is made. Efforts should be made to reduce oxidative stress by using antioxidant supplements and nutritional repletion.
\end{abstract}

Keywords: Chronic renal failure (CRF), Oxidative stress, Serum Nitric oxide, Urea, Creatinine, Estimated glomerular filtration rate (eGFR)

\section{Introduction}

Chronic renal failure is an irreversible deterioration in renal function, which classically develops over a period of years (1). Initially, CRF manifests only as biochemical abnormality. Thereafter, loss of excretory, metabolic and endocrine functions of kidney leads to the chronic renal failure. The diseases that bring about the alterations are many and include diabetes mellitus, hypertension, non-diabetic glomerular disease, poly cystic kidney disease and tubule-interstitial disease (2).Chronic renal failure is associated with premature atherosclerosis andincreased incidence of cardiovascular morbidity and mortality. Several factors contribute to atherogenesis and cardiovascular disease in patients with CRF. Notable among the CRF-induced risk factors are lipid disorders, oxidative stress, inflammation, physical inactivity, anaemia, hypertension, vascular calcification, endothelial dysfunction, and depressed nitric oxide availability $(3,4,5)$.

Hence we thought it worthwhile to determine the extent of dyslipidaemia, theoxidant-antioxidant status and vascular inflammation in various stages of CRF. The present study aimed to assess the levels of serum Urea, Creatinine, superoxide dismutase, lipid peroxidation and Nitric Oxide levels in patients with chronic renal failure.

\section{Material And Methods}

The present study was carried out in the Department of Biochemistry, MNR Medical College and Hospital, Sangareddy. The study protocol was approved by Institutional Ethical Committee of MNR Medical College. The study contains fifty CRF patients and fifty age, sex matched control subjects. Depending on the estimate glomerular filtration rate (eGFR) and confirmed CRF patients were included. Patients who were not willing to participate, had undergone anti-microbial\& anti-inflammatory therapy, used immunosuppressive drugs, with acute inflammatory illness were excluded from the study. According to the eGFR, all CRF patients were subdivided in to five phases and each phase includes $10 \mathrm{CRF}$ patients. Phase I contains Complications in urine sample but healthy kidney function (eGFR >90), Phase II includes Mild renal functional alteration (eGFR 60-90), Phase III includes Moderate renal functional alteration (eGFR 30-60), Phase IV includes Severe renal functional alteration (eGFR 15-30), Phase V about to renal failure (eGFR $<15$ ).

After having deliberate consent from patient, clinical data was collected and $5 \mathrm{ml}$ of venous blood was drawn aseptically and serum was separated. The following parameters were estimated various biochemical methods such as serum Urea by DAM method (6), serum Creatinine by Jaffe's method (7), Superoxide Dismutase by the method of Marklund and Marklund $(8,9)$, Nitric oxide by the method of Najwa K. Cortas and Nabil W. Waked (10). 


\section{Results}

The present study was conducted in Department of Biochemistry, MNR Medical College, Sangareddy during July 2016 to January 2017. The study contains fifty CRF patients and fifty age, sex matched control subjects.

Table1. The mean values of Serum Urea in different phases of CRFpatients and control subjects.

\begin{tabular}{|c|c|c|}
\hline phases of CRF & Number & $\begin{array}{c}\text { Serum Urea (mg/dL) } \\
\text { Mean } \pm \text { SD }\end{array}$ \\
\hline 1 & 10 & $61.42 \pm 16.02$ \\
\hline 2 & 10 & $76.86 \pm 23.71$ \\
\hline 3 & 10 & $97.92 \pm 42.90$ \\
\hline 4 & 10 & $108.31 \pm 40.27$ \\
\hline 5 & 10 & $127.98 \pm 8.97$ \\
\hline Control & 50 & $24.82 \pm 6.51$ \\
\hline
\end{tabular}

Fig 1. Mean values of serum creatinine in different phases of CRF patients and control subjects.

\section{Serum Creatinine in CRF patients and controls}

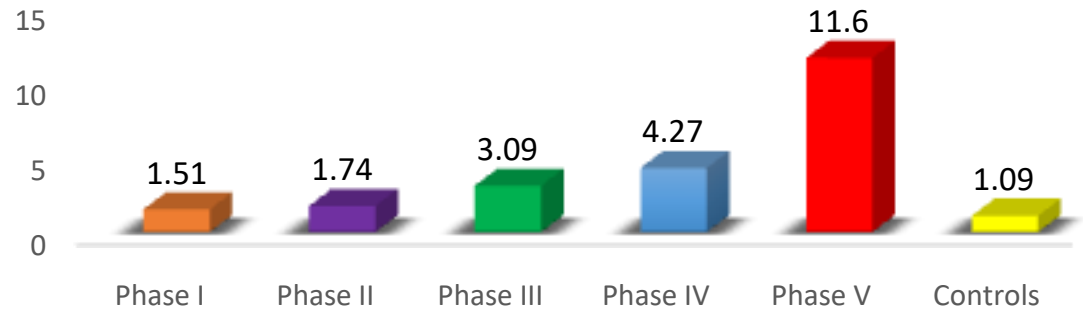

Table 2. The mean values of Serum Lipid peroxidation, Serum Superoxide Dismutase and NitricOxide at various phases of CRF Patients and controlsubjects.

\begin{tabular}{|c|c|c|c|c|}
\hline $\begin{array}{c}\text { Phases } \\
\text { of CRF }\end{array}$ & Number & $\begin{array}{c}\text { Lipid } \\
\text { Peroxidation } \\
(\text { Mean }+ \text { SD }) \\
\text { nmol/mL }\end{array}$ & $\begin{array}{c}\text { Superoxide } \\
\text { Dismutase } \\
(\text { Mean }+ \text { SD }) \\
\text { U/mL }\end{array}$ & $\begin{array}{c}\text { Nitric oxide } \\
\text { (Mean +SD) } \\
\mu \mathrm{mol} / \mathrm{L}\end{array}$ \\
\hline 1 & 10 & $3.14 \pm 0.48$ & $4.59 \pm 1.02$ & $56.27 \pm 3.37$ \\
\hline 2 & 10 & $3.37 \pm 0.42$ & $3.98 \pm 1.25$ & $53.93 \pm 3.72$ \\
\hline 3 & 10 & $5.29 \pm 0.61$ & $3.67 \pm 0.91$ & $51.68 \pm 4.21$ \\
\hline 4 & 10 & $6.21 \pm 0.71$ & $2.12 \pm 0.62$ & $49.07 \pm 3.62$ \\
\hline 5 & 10 & $7.57 \pm 0.70$ & $1.61 \pm 0.59$ & $47.04 \pm 2.98$ \\
\hline Control & 50 & $2.59 \pm 0.49$ & $4.74 \pm 1.11$ & $55.58 \pm 4.69$ \\
\hline
\end{tabular}

The results of above table (Table 2) indicating that serum lipid peroxidation was significantly increased in all the phases of CRF patients when compared to the controls. In related to Serum superoxide dismutase mean values are not showing variation in first phase of CRF and controls, later phases are values were gradual decline in CRF patients as compared to control subjects. Hence, significant increased values was observed in later phases of CRF patients.

\section{Discussion}

Imbalance between production of reactive oxygen species (ROS) and antioxidant defenseleads to oxidative stress, which may arise either from deficiencies of antioxidants or increased formation of ROS such as peroxynitrite, hypochlorous acid or superoxide anions (11).

High levels of oxidative stress markers such as F2-isoprostanes, advanced glycosylation endproducts, malondialdehyde and oxidized LDL have been demonstrated in patients with mild to moderate CRF patients (12). Oxidative modification of LDL is thought to be a key step in the initiation of atherosclerosis (13). Therefore, one may consider the oxidative stress hypothesis as a unifying concept of increased CVD risk in CRF patients (14).Superoxide dismutase (SOD) functions as an antioxidant by scavenging superoxide anion which is 
derived from molecular oxygen by single electron transfer. SOD converts the highly reactive superoxide radical into less toxic hydrogen peroxide and decreases cell damage. The lower serum SOD levels point towards the deficient antioxidant mechanisms in chronic kidney disease (15).

In the present study the serum superoxide dismutase (SOD) was found to be lower in patients at all the phases of chronic renal failure. Nitin et al and Sasikala et al have been reported similar reduction in SOD levels in CKD patients $(16,17)$. In the present study, significant reduction of SOD levels at all phases correlating with the study by Nitin et al where SOD levels found to be highly significant in all stages of CRF patients (16).

Serum lipid peroxidation was significantly increased in all the phases of CRF patients when compared to the controls. Serum nitric acid levels were higher in first stage as compared to control subjects but significantly decreased in succeeding phases i.e. phase II to Phase V. Nitric oxide is a key factor in the antiatherosclerotic effects of endothelium (18). Premature atherosclerosis is one of the primary cause of morbidity and mortality in patients with CRF (19). Hyperhomocysteinemia, oxidative stress and endothelial dysfunction may be interrelated, forming a cascade of athero-thrombotic processes in CRF.

\section{Conclusion}

Chronic renal failure is a progressive loss in renal function over a period of time. This study aimed to assess the oxidative stress, serum nitric acid, Urea, creatinine and nitric oxide levels in CRF patients. The results concluding that oxidative stress markers are increasing, while superoxide dismutase levels were decreased, which presides into endothelial dysfunctionalong with nitric oxide levels reduction. It is extremely important to know oxidative stress in CRF patient's right from the time of diagnosis is made. Efforts should be made to reduce oxidative stress by using antioxidant supplements and nutritional repletion.

\section{Acknowledgements: No}

\section{References}

[1]. Jha V, Garcia-Garcia G, Iseki K, Li Z, Naicker S, Plattner B, Chronic Kidney Disease: global dimension and perspectives, Lancet, 382(9888), 2013,260-72.

[2]. Haslett C, Chilvers ER, Hunter A, Boon NA. edn, In: Davidson's Principle and Practice of Medicine, 18th edi. UK. 1999, 433-39.

[3]. Vaziri ND, Oxidative stress in uremia. Nature Mechanisms and Potential consequences, Seminars in nephrology, 2004, 469-73.

[4]. Zalba G, Fortuno A, Diez J, Oxidative stress and atherosclerosis in early chronic disease, Nephrology Dialysis Transplantation, 21(10), 2006, 2686-2690.

[5]. Luciak M, Antioxidants in the treatment of patients with renal failure,AnnalesAcademiaeMedicaeBialostocensis, 49, $2004,157-161$.

[6]. Marsh WH, Fingerhut B, Miler H, Automated and manual direct methods for determination of blood urea, Clin Chem, 11, 1965, 624-627.

[7]. Fossati P, Ponti M, Passoni G, Tarenghi G, Melzid'Eril GV, Prencipe L, A Step forward in enzymatic measurement of creatinine, Clin Chem,40, 1974, 130-137.

[8]. Marklund S, Marklund G, Involvement of the superoxide anion radical in the autoxidation of pyrogallol and a convenient assay for superoxide dismutase,Eur J Biochem, 47, 1974, 469-474.

[9]. Nischal HK, Sharma MP, Goyal RK, Kaushik GG, Serum superoxide dismutase levels in diabetes mellitus with or withoutmicroangiopathic complications, JAPI, 46, 1998, 853-855.

[10]. Cortas NK, Wakid NW, Determination of inorganic nitrate in serum and urine by a kinetic cadmimum method, Clin Chem, 36(8), 1990, 1440-1443.

[11]. Halliwell B, The role of oxygen radicals in human disease, with particular reference to the vascular system, Haemostasis, 23(Suppl1), 1993, 118-26

[12]. Cachofeiro V, Goicochea M, de Vinuesa SG, Oubina P, Lahera V, Luno J, Oxidative stress and inflammation, a link between chronic kidney disease and cardiovascular disease, Kidney Int, 111(Suppl) 2008, S4-S9.

[13]. Steinberg D, Parthasarathy S, Carew TE, Khoo JC, Witztum JL, Beyond cholesterol. Modifications of low-density lipoprotein that increase its atherogenicity, N Engl J Med, 320, 1989, 915-24.

[14]. Himmelfarb J, Stenvinkel P, Ikizler TA, Hakim RM, The elephant in uremia: oxidant stress as a unifying concept of cardiovascular disease in uremia, Kidney Int, 62, 2002, 1524-38.

[15]. Annuk M, ZilmerM, Lind L, Linde T, Fellstrom B, Oxidative stress and endothelial function in chronic renal failure, J Am SocNephrol, 12, 2001, 2747-2752.

[16]. Nagane NS, Ganu JV, Gandhi R, Oxidative stress, serum homocysteine and serum nitric oxide in different stages of chronic renal failure, Biomedical Research, 20(1), 2009, 71-4.

[17]. Sasikala M, Subrahmanyam C, Sadasivadu, Early oxidative change in low density lipoproteins during progressive chronic renal failure, Indian J Clini Biochem, 14(2), 1994, 176-83.

[18]. Nagane NS, Ganu JV, Gandhi R, Oxidative stress, serum homocysteine and serum nitric oxide in different stages of chronic renal failure, Biomedical Research, 20(1), 2009, 71-4.

[19]. Halliwell B, Gutteridge JMC, Free Radicals in Biology and Medicine, $3^{\text {rd }}$ edn, Oxford, Oxford University Press, 1999, $140-163$. 